\title{
Rare scar sarcoidosis: A case report
}

\author{
SHA ZHAO, QIN WANG, BEIBEI CHENG and XIAO-FANG ZHU
}

Department of Dermatology, Clinical Medical School of Yangzhou University, Yangzhou, Jiangsu 225001, P.R. China

Received July 27, 2015; Accepted December 1, 2016

DOI: $10.3892 /$ etm.2017.4123

\begin{abstract}
A 70-year-old female patient presenting with swelling and subcutaneous nodule formation localized to the knee scars over a two-month period is presently reported. Dermatological examinations found erythematous swelling and small papules on the patient's knee scars, along with subcutaneous nodules beneath and around the scars. Computer tomography of the chest region identified multiple obscure tubercles in the lungs and swollen lymph nodes in the hilum pulmonis and mediastinum. Skin biopsy revealed multiple non-caseating granulomas, which had infiltrated the dermis. The patient was diagnosed with scar sarcoidosis as a result of these observations. The onset of scar sarcoidosis is rare and, therefore, not well understood; however, inflammatory alterations in preexisting scars may be important indicators for disease onset.
\end{abstract}

\section{Introduction}

Sarcoidosis is a systemic inflammatory disorder of unknown etiology, which is characterized and defined by the presence of non-caseating granulomata. The incidence of this multisystem disease is 10-15 cases per 100,000 each year (1). The development of sarcoidosis has been associated with a number of environmental factors, genes, immune factors, and exposure to certain microbial agents (1). Approximately $25 \%$ of sarcoidosis may have cutaneous involvement, with a variety of clinical presentations (2) and pulmonary involvement occurs in $90-95 \%$ of cases. Therefore patients with cutaneous sarcoidosis require an evaluation of symptoms that may be caused by extracutaneous diseases (1). Among cutaneous sarcoidosis, scar sarcoidosis is rare and occurs predominantly in young adults, particularly in females (2). The present study reports the case of an elderly female patient who developed scar sarcoidosis in 20-year-old scars initially acquired in a traffic accident.

Correspondence to: Dr Xiao-Fang Zhu, Department of Dermatology, Clinical Medical School of Yangzhou University, 98 Nantong West Road, Yangzhou, Jiangsu 225001, P.R. China

E-mail: sharonzhu66@126.com

Key words: sarcoidosis, scar, knee

\section{Case report}

A 70-year-old female patient presented painful reactivation of 20-year-old-scars located on the knees, originally acquired in a traffic accident. The patient suffered localized scar pain without itching for two months prior to her visit to the outpatient dermatology clinic at the Dermatology Department of the Clinical Medical School of Yangzhou University on 12th October 2012. Systemic symptoms such as weight loss, fever and cough were also absent and systematic physical examinations for the respiratory, cardiovascular and nervous systems, such as lung and heart auscultation, reported normal results. However, dermatological physical examinations found erythematous swelling and small papules localized to the knee scars and, on the right knee, two subcutaneous indurated nodules, one $3 \times 5 \mathrm{~cm}$ in size and another one $2 \times 3 \mathrm{~cm}$ in size, were found under and below the scars, respectively (Fig. 1A).

The patient additionally underwent auxiliary examinations, including a complete blood count and tests to determine erythrocyte sedimentation rate, c-reactive protein and electrolyte levels, and liver and renal function, which identified no pathological findings. Blood levels of angiotensin-converting enzyme, typically used to aid in the diagnosis of sarcoidosis (2), was 52 IU/1 (reference range 24-65 IU/1), and the urinary analysis was found to be normal. The tuberculosis skin test also reported negative results (the size of red nodule in test site was $>5 \mathrm{~mm}$ ). However, a biopsy was carried out on the skin lesion and histopathology under light microscopy (Olympus CX41; Olympus Corporation, Tokyo, Japan) of the tissue revealed multiple non-caseating granulomas composed of epithelioid cells, numerous multinucleated giant cells and a small number of lymphocytes (Fig. 1B and C).

The patient's chest was also examined. A chest $x$-ray revealed bilateral hilar lymphadenopathy (Fig. 2A), while computer tomography of the chest revealed multiple obscure tubercles (largest one $\sim 10 \mathrm{~mm}$ in size) in the lungs and swollen lymph nodes in the hilum pulmonis and mediastinum (Fig. 2B and C). Following the diagnosis of scar sarcoidosis, the patient refused treatment. The patient provided informed written consent for her data to be used in the present study.

\section{Discussion}

Sarcoidosis is a systemic disease with an unknown etiology. The disease is characterized by the formation of epithelioid granulomas that lack caseations, with skin lesions occurring in $20-25 \%$ of cases (2). The diversity of clinical presentations 

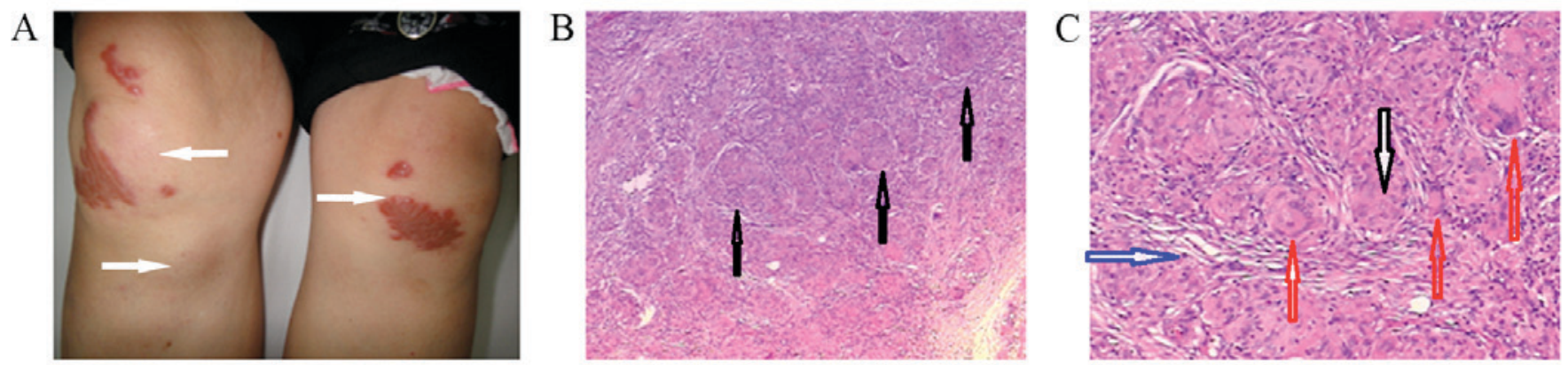

Figure 1 (A) The patient's knee scars with swelling and nodules. Lesions on the right knee displayed subcutaneous nodules beneath and around the scars (white arrow). Lesions on the left knee displayed swelling (white arrows). (B and C) Histopathological features of the scar tissue. (B) Multiple non-caseating granulomas (black arrows) were found infiltrating the dermis and subcutis (hematoxylin and eosin staining, magnification x40). (C) Granulomas were composed of epithelioid macrophages (black arrow), multinucleated giant cells (red arrows) and a small number of lymphocytes (blue arrow; hematoxylin and eosin staining, magnification x100).
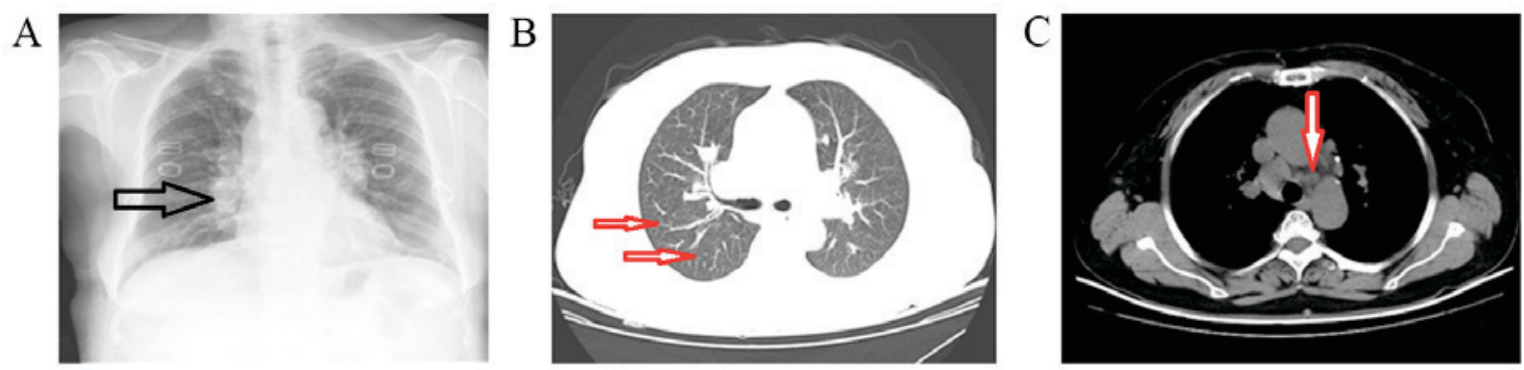

Figure 2. Patient chest examinations. (A) Chest x-ray displaying bilateral hilar enlargement (black arrow). (B and C) Chest computer tomography revealing (B) multiple obscure tubercles on the lungs (red arrows) and (C) swollen lymph nodes in mediastinum (red arrow).

mean that sarcoidosis is difficult to diagnose. Disease diagnosis requires a combination of: i) Supporting clinical-radiological findings such as hilar and/or paratracheal lymph node enlargement with or without pulmonary infiltrates; ii) histological evidence of non-caseating epithelioid granulomas at disease sites; and iii) exclusion of alternative causes for the granulomatous inflammation and local sarcoid-like reactions (3). Skin lesions in sarcoidosis may appear as maculae, papules, plaques, nodules, ulcers, localized alopecia, ichthyotic areas, subcutaneous nodules, lupus pernio, scar sarcoidosis, psoriasiform and even pustules. Among these, scar sarcoidosis is rare, accounting for $5.4-13.8 \%$ of sarcoidosis cases $(4,5)$. The present case of scar sarcoidosis is the third to be recorded in China $(6,7)$.

Scar sarcoidosis is characterized by the onset of erythematous swelling and the development of papules and nodules within the original scars. In cases of cutaneous or subcutaneous swelling in the area of an old scar or beside a scar, a scar sarcoidosis is a possible differential diagnosis (1). Excluding skin damaged by mechanical injury, scar sarcoidosis can occur on skin sites damaged by a range of factors, including venipuncture, intramuscular injections, inoculations, tattoos and infections such as herpes zoster (8). It is suggested that foreign material within the scar, deposited by external factors including those stated above, is a possible cause of epithelioid granuloma (9). The specific skin lesions that occur and the resulting sarcoidosis may be associated with the severity and duration of the disease, with scar sarcoidosis often being accompanied by systemic involvement (4). Alterations, such as further damage or stress to the existing scars, often prompt worsening of sarcoidosis (10).
The case of scar sarcoidosis presented here is an elderly woman, displaying no other health problems, including tuberculosis and hepatitis. The patient visited the clinic due to the development of painful nodules and erythematous swelling on preexisting scars located on her knees, which formed following a traffic accident 20 years prior. Although the patient did not report any systemic symptom, physical examinations found that she had stage II pulmonary involvement in the sarcoidosis, while all other organs were not involved. As chest X-ray or computed tomography scan examination was not performed on the patient within several years, it could not be determined whether the onset of pulmonary involvement occurred before or after scar sarcoidosis; however the majority of patients with scar sarcoidosis develop systemic disease (8). Therefore, it is necessary for patients who are presenting with painful inflammation on or around their existing scars to be examined systematically and followed up. Standard therapies for sarcoidosis include the administration of corticosteroids, antimalarials and methotrexate (11). However, scar sarcoidosis often resolves slowly and spontaneously (4). Thus, the patient in the present case refused treatment. Alternative second-line drug treatments include methotrexate and hydroxychloroquine; however, these agents are not $100 \%$ effective (12). For patients with progressive cutaneous sarcoidosis or refractory cases, monoclonal antibodies are a novel therapeutic option. For example, etanercept antibodies $(3,13)$ that target tumor necrosis factor- $\alpha$ have been demonstrated to be beneficial in treating recalcitrant sarcoidosis. In the treatment of scar sarcoidosis, injection of the corticosteroid triamcinolone acetonide into the skin lesions is 
also effective (8). Furthermore, it is necessary to continually survey patients via pulmonary examination and chest radiography every two months, as well as periodic monitoring for other systemic manifestations (8).

Unfortunately, a follow-up of the patient in the present case was not possible. Contact with the patient was attempted on numerous occasions; however the contact information provided was invalid.

\section{References}

1. Henrichs MP, Streitbürger A, Gosheger G, Surke C, Dierkes C and Hardes J: Scar sarcoidosis on a finger mimicking a rapidly growing soft tissue tumour: A case report. BMC Res Notes 5: $545-549,2012$

2. Fernandez-Faith E and McDonnell J: Cutaneous sarcoidosis: Differential diagnosis. Clin Dermatol 25: 276-287, 2007.

3. Spagnolo P: Sarcoidosis: A critical review of history and milestones. Clin Rev Allergy Immunol 49: 1-5, 2015.

4. Marchell RM and Judson MA: Chronic cutaneous lesions of sarcoidosis. Clin Dermatol 25: 295-302, 2007.
5. Mana J, Marcoval J, Graells J, Salazar A, Peyrí J and Pujol R: Cutaneous involvement in sarcoidosis. Relationship to systemic disease. Arch Dermatol 133: 882-888, 1997.

6. Shen B, Chen XL and Wu LM: Scar sarcoidosis: First case report in China. Chin J Dermatol 44: 91-93, 2011.

7. Zeng YP, Jiang GT, Qu T, Ma DL, Liu YH, Jin HZ and Sun QN: A case report of Scar sarcoidosis. Chin J Dermatol 44: 211-212, 2011 (In Chinese).

8. Choi SY, Hyun MY, No YA, Park KY, Li K, Kim BJ, Seo SJ, Kim MN and Hong CK: Scar sarcoidosis on a hypertrophic scar. Clin Exp Dermatol 39: 945-947, 2014.

9. Cervigon I, Palomo A and Torres LM: Cutaneous lesions on scars. Actas Dermosifiliogr 98: 433-434, 2007 (In Spanish).

10. Marcoval J, Mañá J and Rubio M: Specific cutaneous lesions in patients with systemic sarcoidosis: Relationship to severity and chronicity of disease. Clin Exp Dermatol 36: 739-744, 2011.

11. Doherty CB and Rosen T: Evidence-based therapy for cutaneous sarcoidosis. Drugs 68: 1361-1383, 2008.

12. Haimovic A, Miguel S, Judson MA and Prystowsky S: Sarcoidosis: A comprehensive review and update for the dermatologist: Part I. Cutaneous disease. J Am Acad Dermatol 66: 699. e1-e18, 2012.

13. Tuchinda $\mathrm{C}$ and Wong HK: Etanercept for chronic progressive cutaneous sarcoidosis. J Drugs Dermatol 5: 538-540, 2006. 